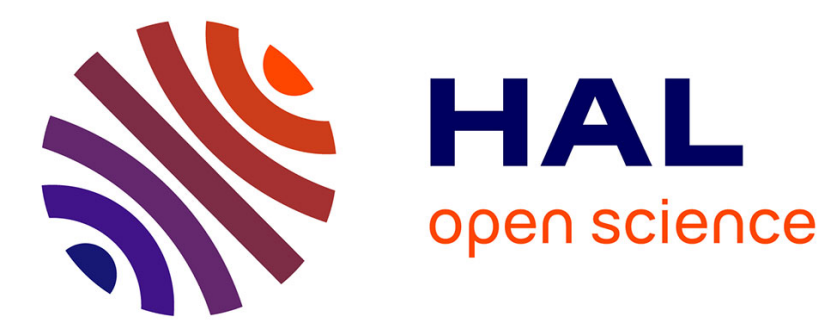

\title{
LHCb calorimeters and muon system lepton identification
}

F. Machefert

\section{To cite this version:}

F. Machefert. LHCb calorimeters and muon system lepton identification. International Conference on B-Physics at Hadron Machines - BEAUTY 2003 9, Oct 2003, Pittsburgh, United States. pp.123-126. in2p3-00021967

\section{HAL Id: in2p3-00021967 https://hal.in2p3.fr/in2p3-00021967}

Submitted on 22 Jun 2004

HAL is a multi-disciplinary open access archive for the deposit and dissemination of scientific research documents, whether they are published or not. The documents may come from teaching and research institutions in France or abroad, or from public or private research centers.
L'archive ouverte pluridisciplinaire HAL, est destinée au dépôt et à la diffusion de documents scientifiques de niveau recherche, publiés ou non, émanant des établissements d'enseignement et de recherche français ou étrangers, des laboratoires publics ou privés. 


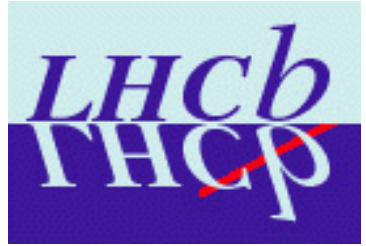

\section{Calorimeter and Muon System}

\section{Lepton Identification}

Pittsburgh, October 14 - 18, 2003

On Behalf of the LHCb Collaboration

Frédéric MACHEFERT 


\section{The LHCb experiment}

$\square$ LHCb is dedicated to the Study of CP violation in the B meson system

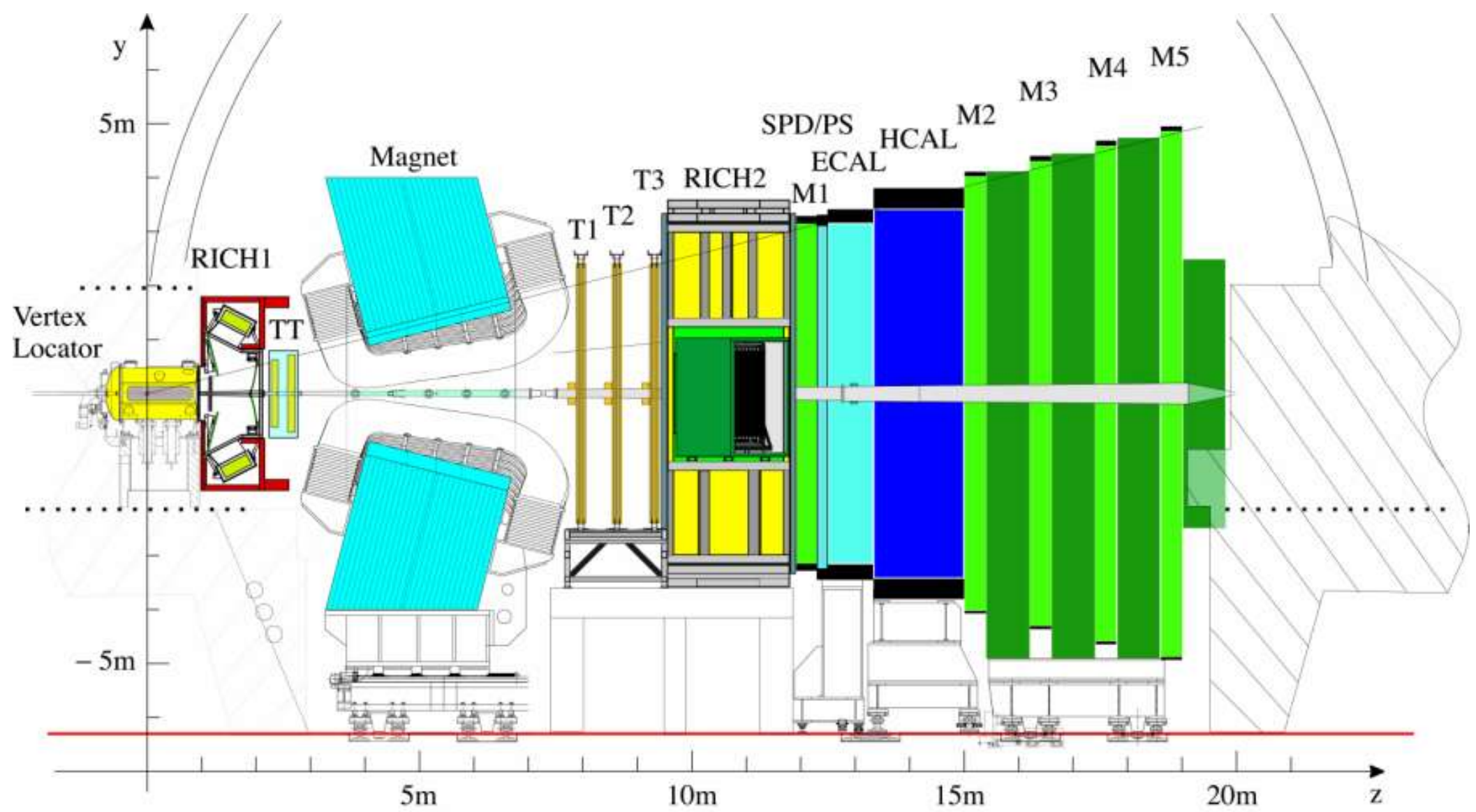

- recent reoptimisation

- VELO, RICH1, Tracking

- Less material before Calorimeter $\left(\sim 70 \% \mathrm{X}_{0}\right)$ 


\section{LHCb Calorimeters}

Requirements :

E Identification of hadrons, electrons, $\gamma, \pi^{0}$

E Energy/ Position measurement

E LO Trigger input : $\rightarrow$ see talk by O. Callot

- High sensitivity

- Fast response $(40 \mathrm{MHz})$

\section{Scintillator Pad Detector (SPD)}

Preshower (PRS) $\rightarrow$ L0

- $\gamma /$ MIP separation (SPD)

- Electron, $\gamma / \pi$ (PRS)

- Charged Multiplicity (SPD)

$\square \mathrm{ECAL} \rightarrow \mathrm{LO}$

E Et of electron, $\gamma$

- $\pi^{0}$ offline reconstruction

$\square \mathrm{HCAL} \rightarrow \mathrm{LO}$

- Et of hadrons

- Particle ID

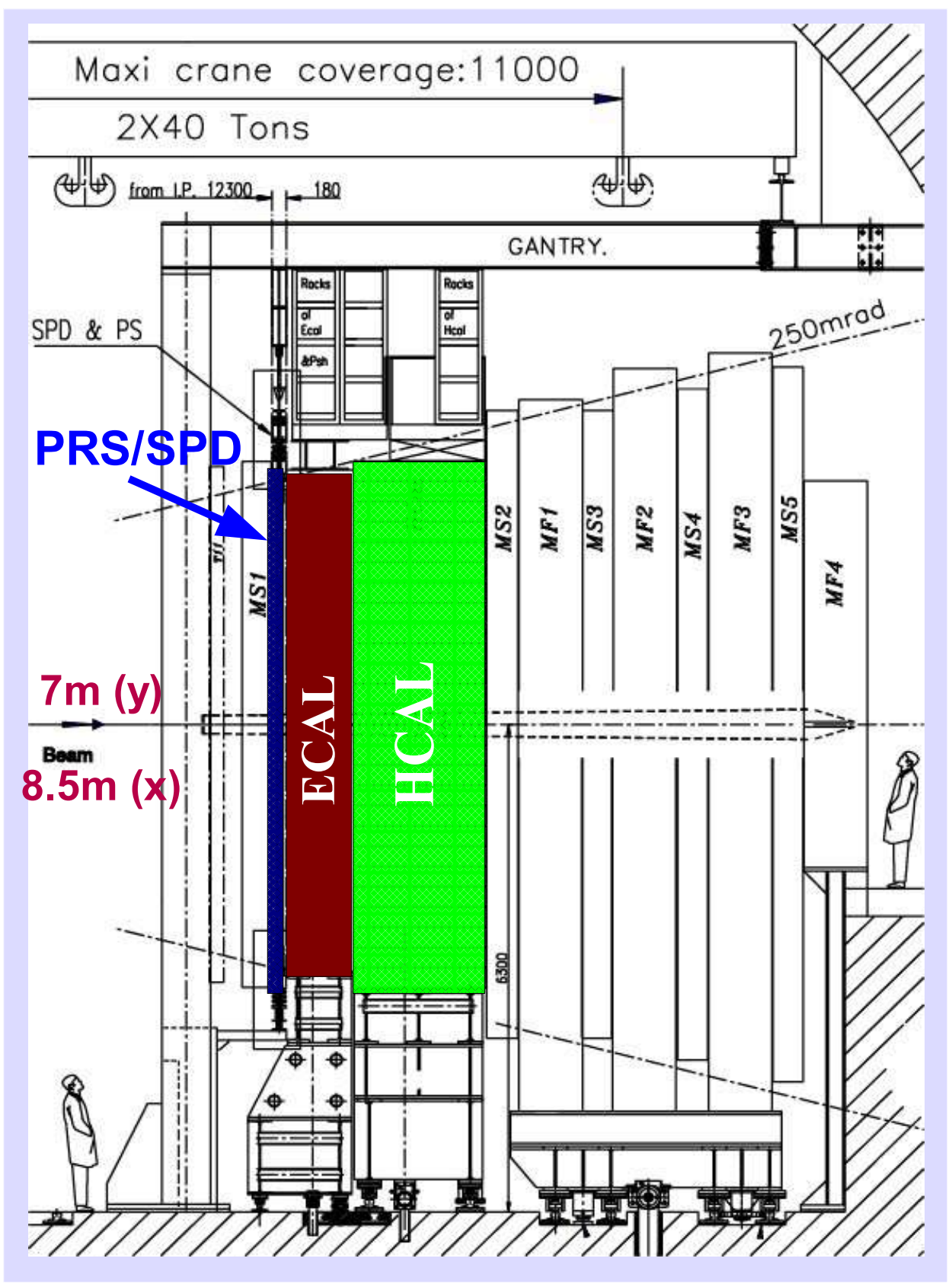




\section{SPD / PRS}
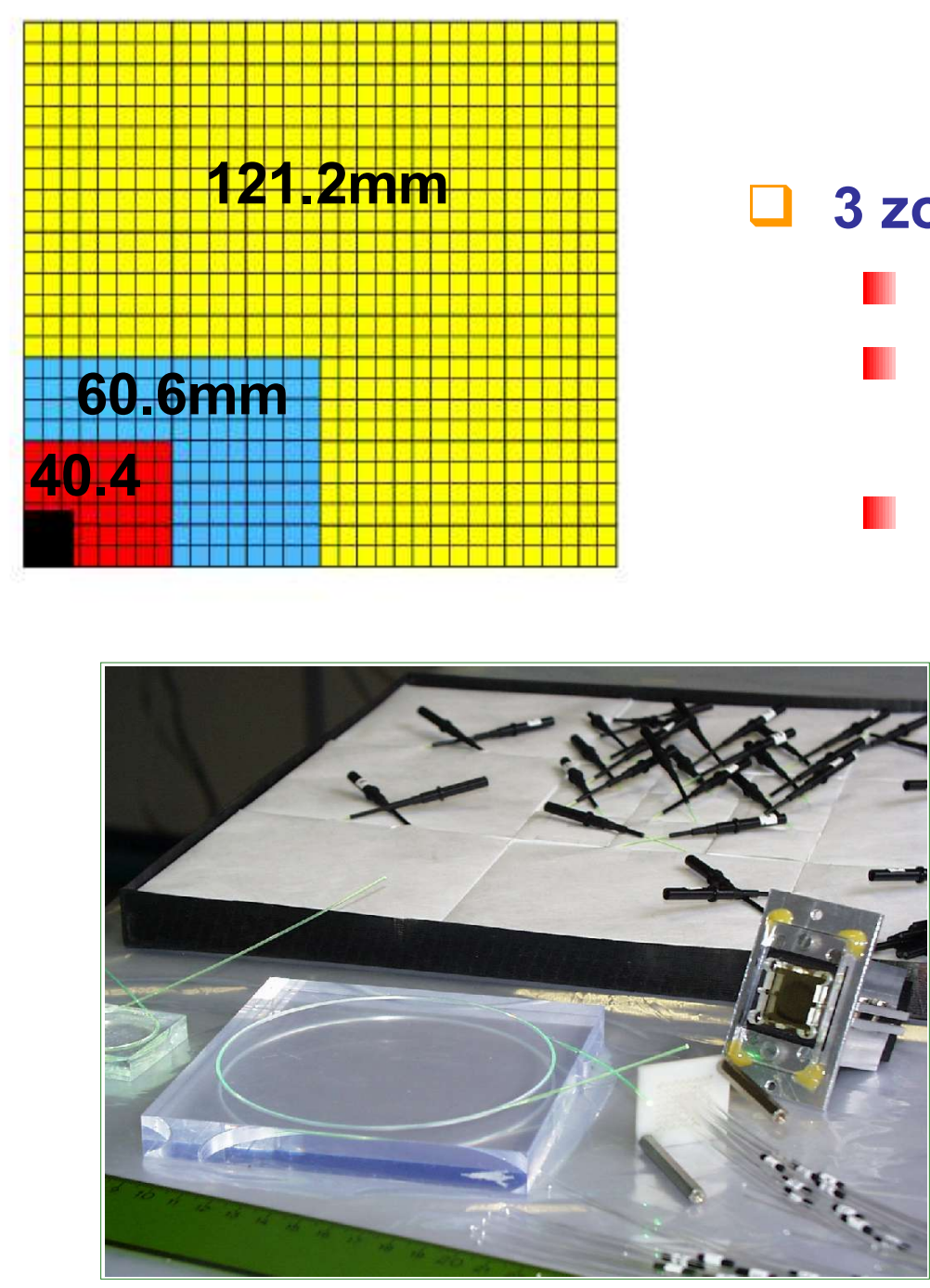

$\square$ Scintillating detector

E $2.5 \mathrm{X}_{0}$ lead converter sandwiched between two scintillator planes (pads)

$\square 3$ zones : granularity depends on the occupancy

E Cell size : 40.4 / $60.6 / 121.2 \mathrm{~mm}$

E $~ 6000$ channels

- Notice : 3 same zones for ECAL (HCAL : 2 zones)

- Projective Calorimeters

$\square$ LO :

- ECAL finds local Et maxima

- SPD/PRS determines electromagnetic nature of energy deposit

$\square$ Signal read with MAPMT

$\square$ Dynamic range : 0 - 100 Mips

E 10 bits (PRS) +1 bit (SPD) 


\section{ECAL}

\section{Shashlik technology}

- Radiation resistance

- Fast response

- 66 layers of $2 \mathrm{~mm} \mathrm{~Pb} / 4 \mathrm{~mm}$ scintillator

- $25 X_{0}, 1.1 \lambda_{1}$

E WLS fibres transport signal to PMT
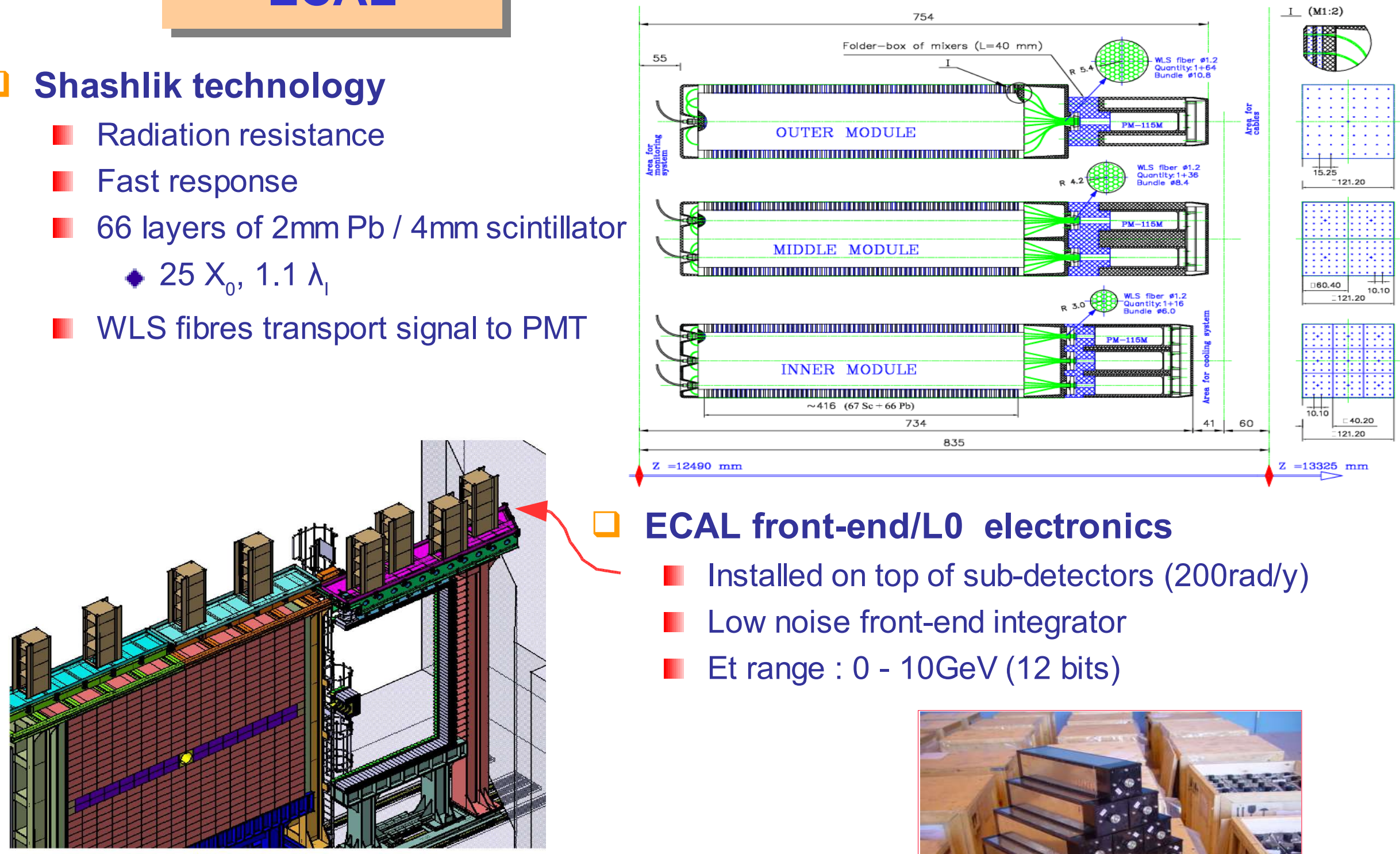

\section{ECAL front-end/L0 electronics}

- Installed on top of sub-detectors (200rad/y)

- Low noise front-end integrator

Et range : 0 - $10 \mathrm{GeV}$ (12 bits)

$\square 90 \%$ of the modules delivered to CERN 


\section{HCAL}

\section{$\square$ Longitudinal tiles}

- Iron and scintillator tiles

- $6 \mathrm{~mm}$ master, $4 \mathrm{~mm}$ spacer / $3 \mathrm{~mm}$ scintillator

- $5.6 \lambda_{1}$

E 2 zones (1468 channels)

- Signal propagates with WLS fibres to PMT

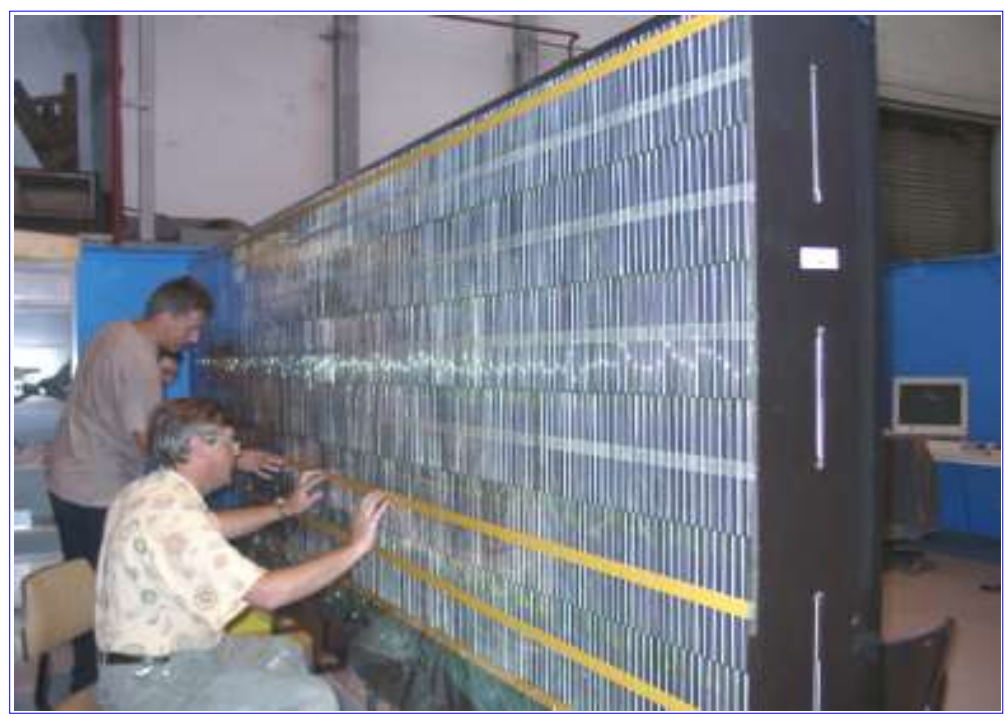

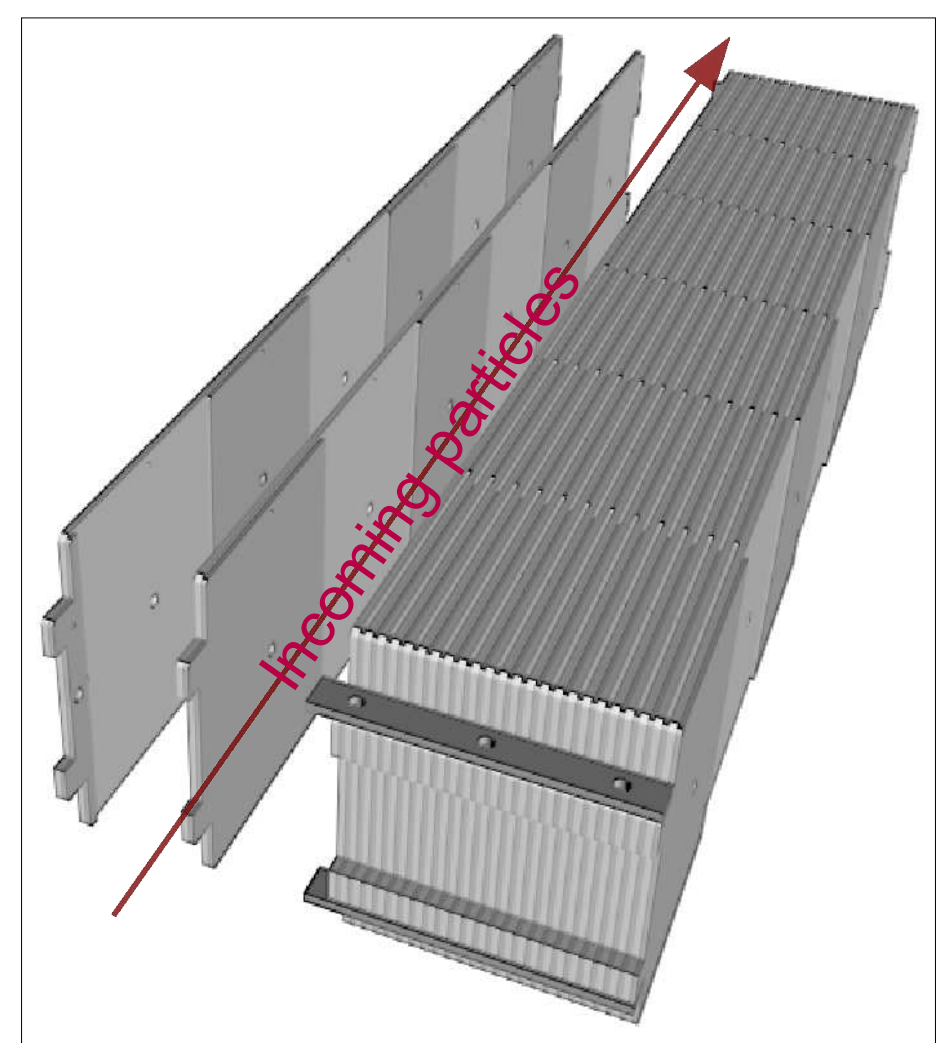

- Same front-end electronics as ECAL

$30 \%$ of the modules already delivered 


\section{ECAL-HCAL Electronics}

$\square$ Scintillator + WLS fibre : fast system

E Try to measure every bunch crossing : no pileup effect (residue after $25 \mathrm{~ns}:<1 \%$ )
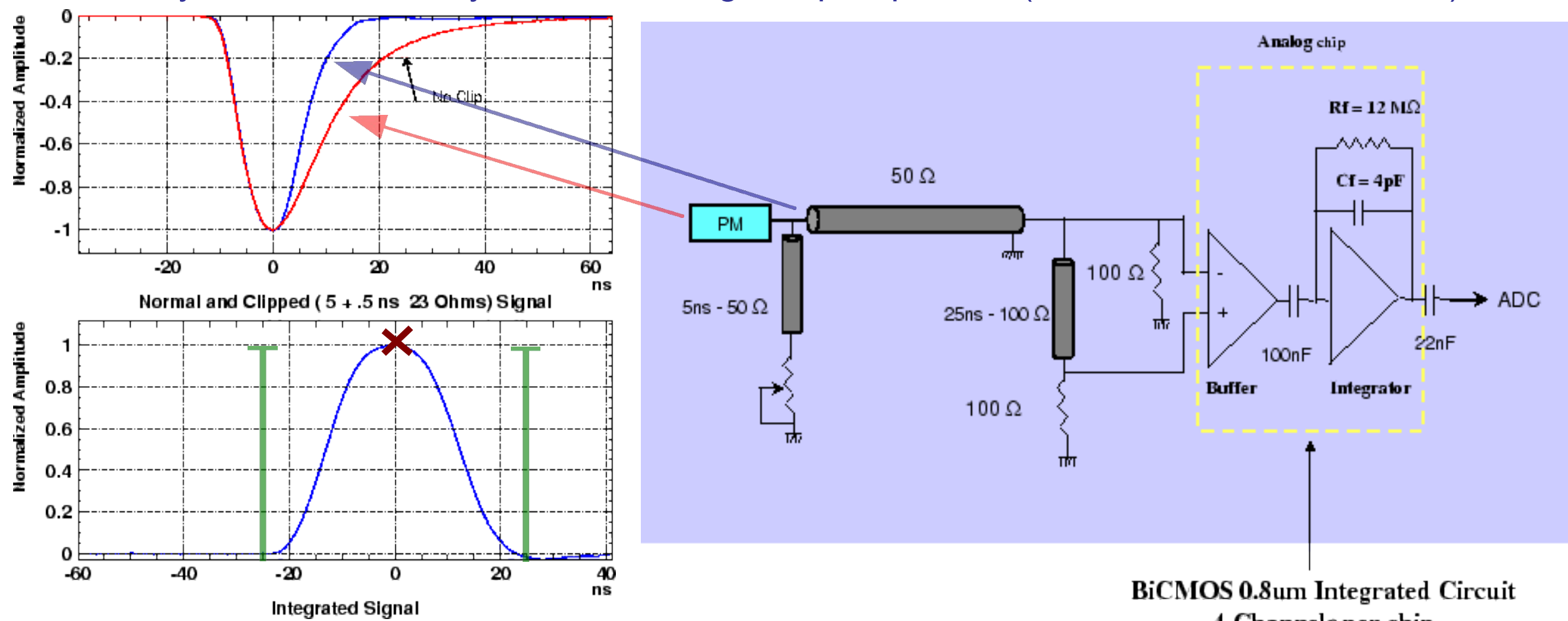

BiCMOS 0.8um Integrated Circuit

4 Channels per chip

$\square$ Digital electronics based on Actel anti-fuse technology

- FPGA configuration insensitive to Single Event Effects

- Protection by Parity code and Triple Voting

$\square$ All components have been tested in proton and ion beams 


\section{Energy Resolution}

Energy Resolution of series modules (test beam measurements)
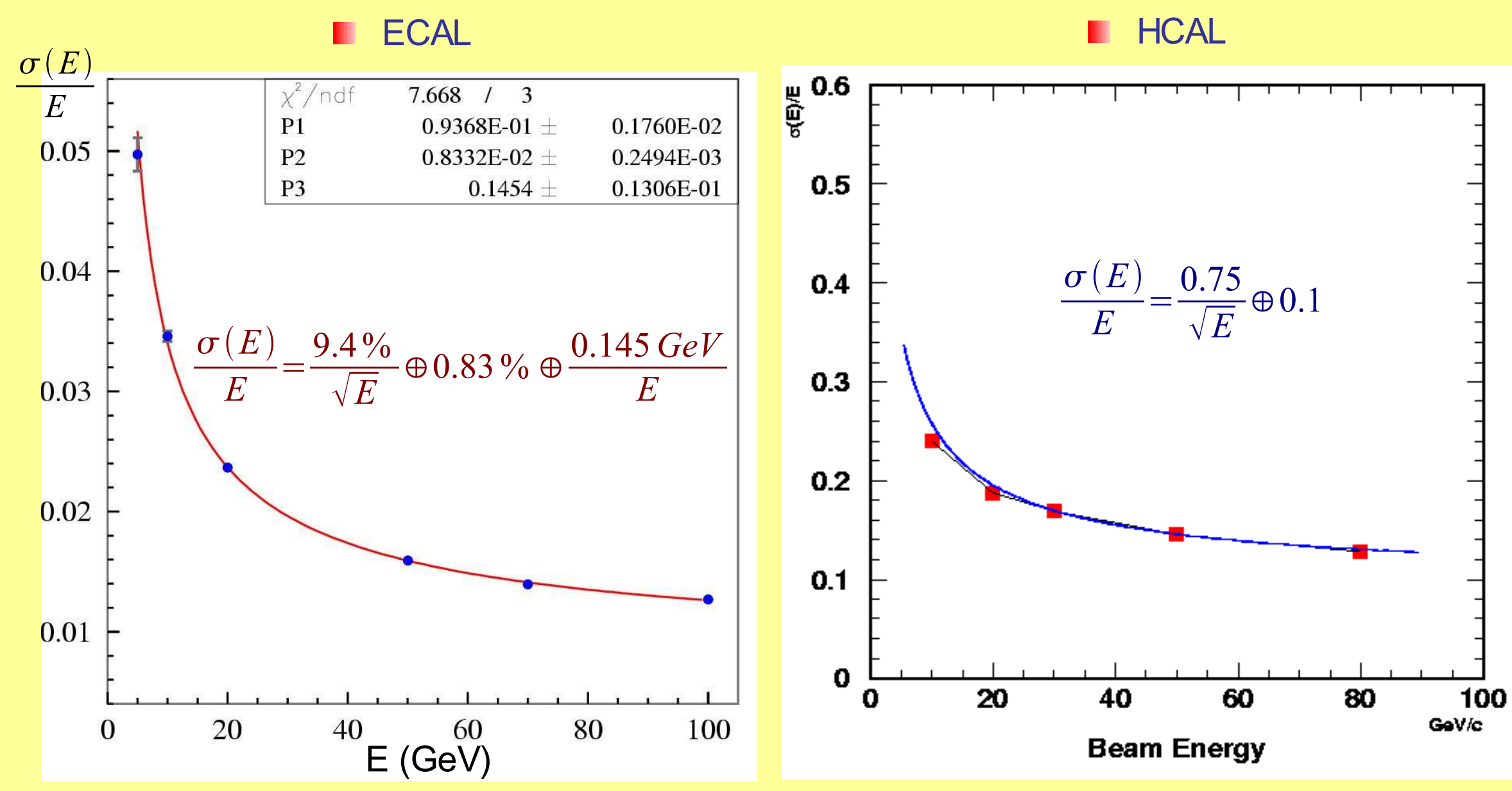


\section{Calorimeter Performances : $\pi^{0}$ reconstruction}

\section{$\pi^{0}$ reconstruction efficiency}

- Resolved $\pi^{0}$ (2 clusters)

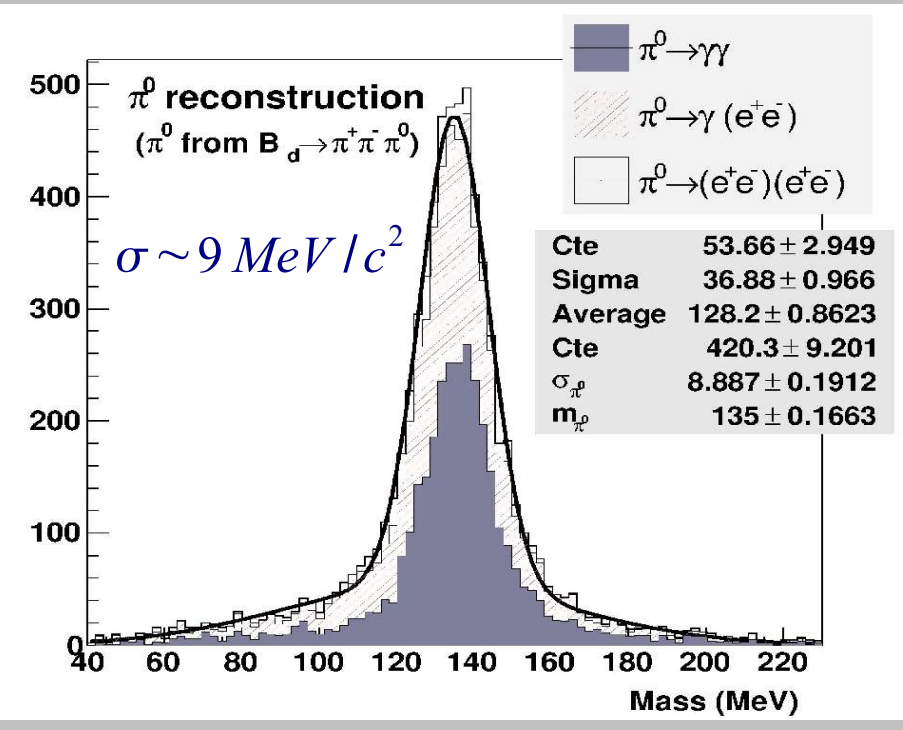

$\pi^{0}$ efficiency inside detector acceptance $\sim 50 \%$

E Merged $\pi^{0}$ ( 1 cluster)
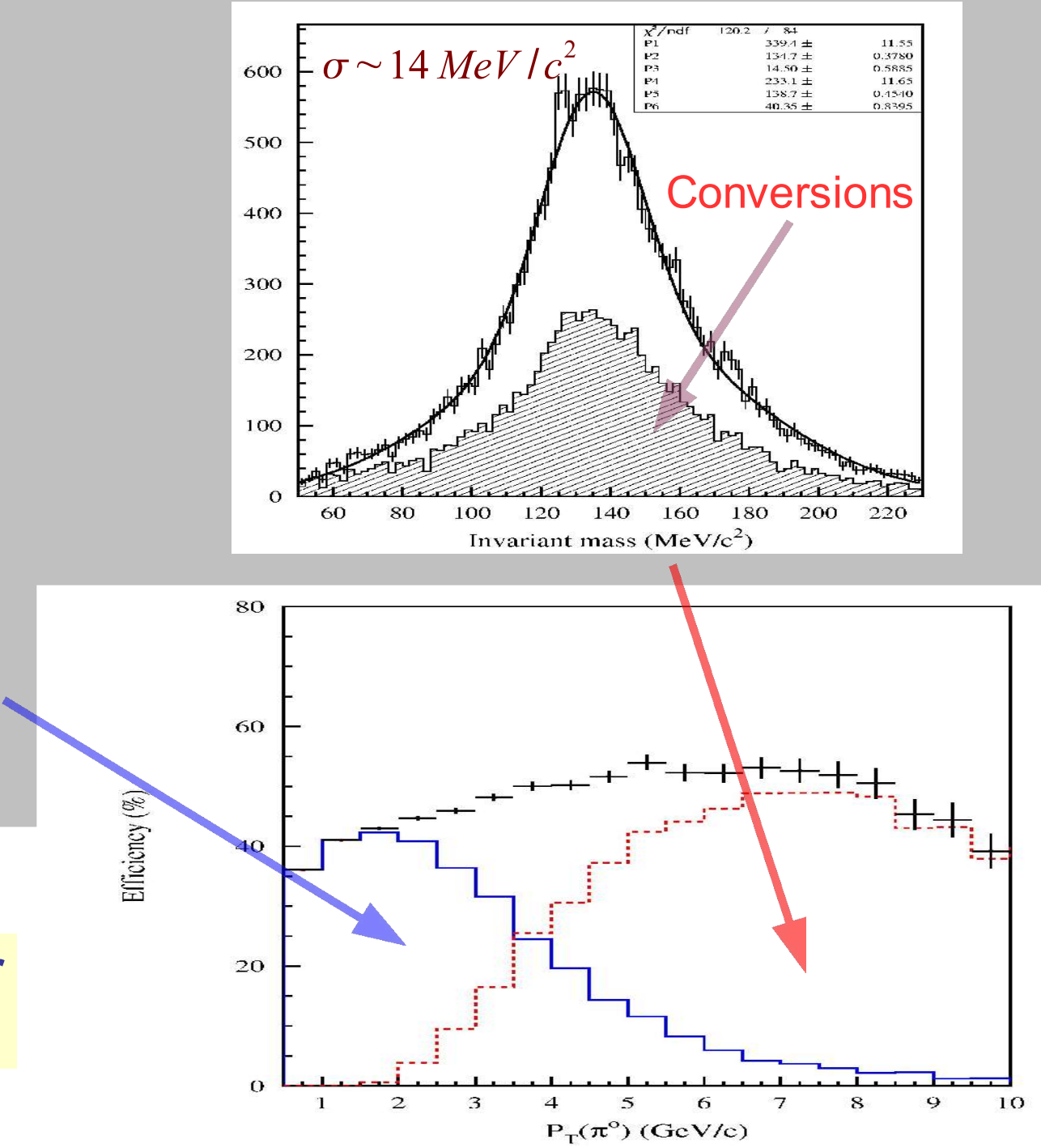


\section{Muon System}

$\square$ Requirements

E Muon triggering (LO)

- Fast measurement / Bunch crossing id.

- High efficiency (down to $p=5 \mathrm{GeV} / \mathrm{c}$ )

- Pt resolution 20\%

- Muon offline identification

- Tagging + reconstruction

- $\varepsilon>90 \%$, mid-id $<1.5 \%$

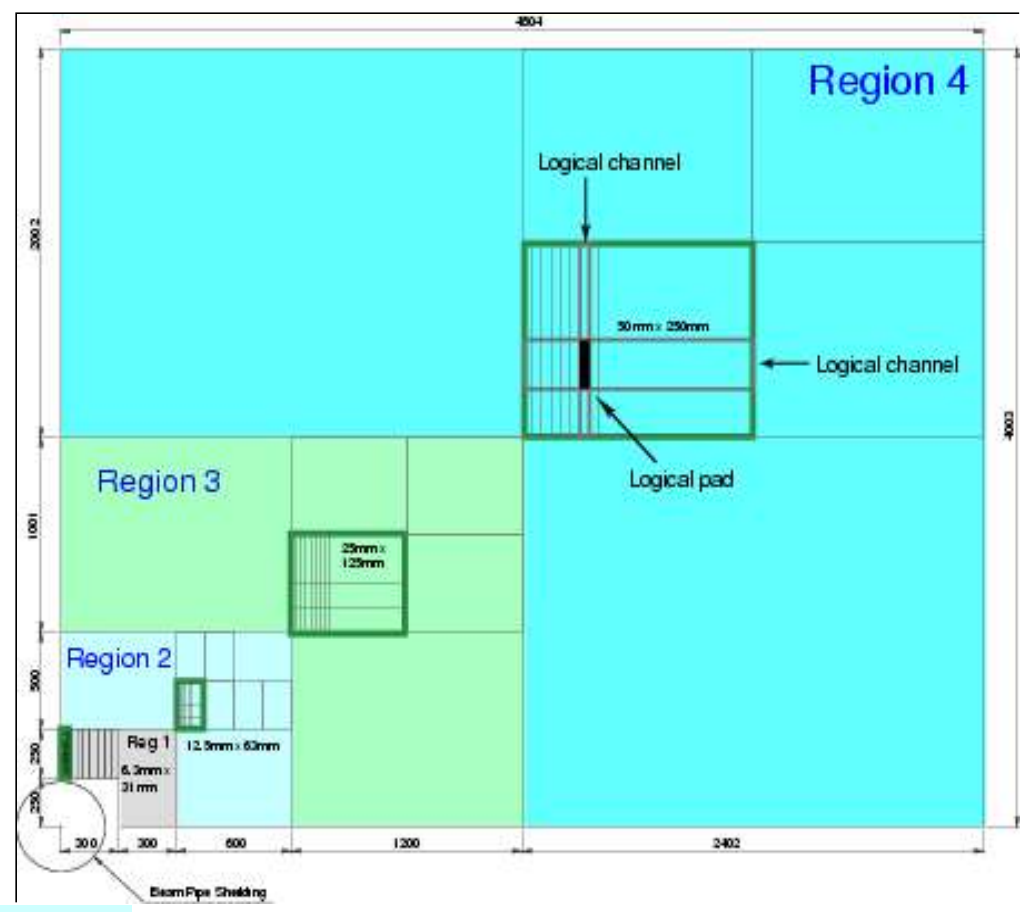

$\square$ Layout

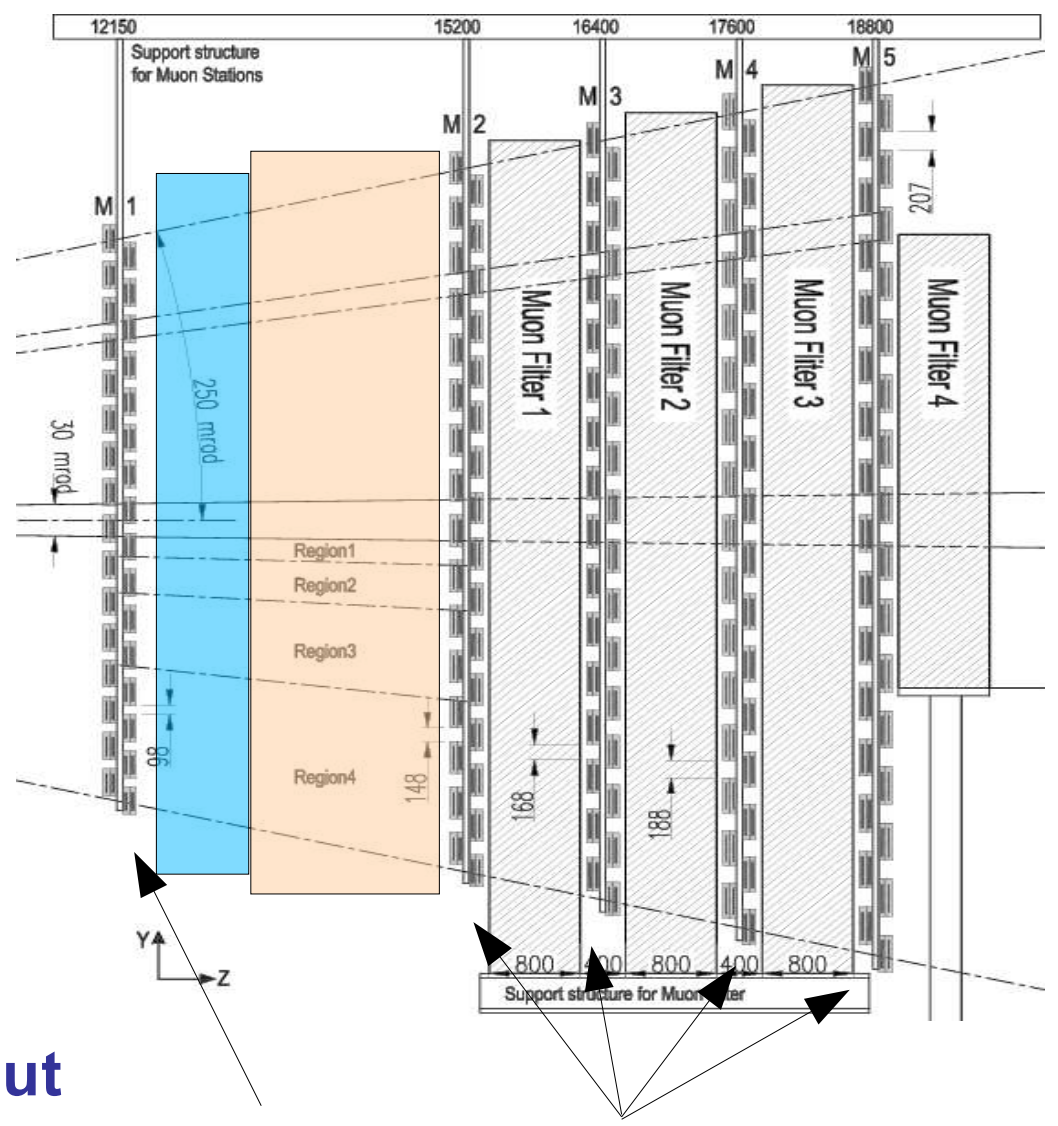

- 5 stations : $1 \times(2$ layers $)+4 \times(4$ layers $)$

- Projective geometry

$\square$ Granularity : Stations divided in 4 regions

E X-dim: L0 pt resolution

E Y-dim: background rejection

- Logical pads from logical channels

$435 m^{2}-1380$ chambers $-26 k$ channels 


\section{MWPC}

\section{MWPC}

- Wire/cathode reading

- $2 \mathrm{~mm}$ wire pitch $\varepsilon=99 \%$ (20ns-2 gaps)

- Time resolution : $4 \mathrm{~ns}$

1

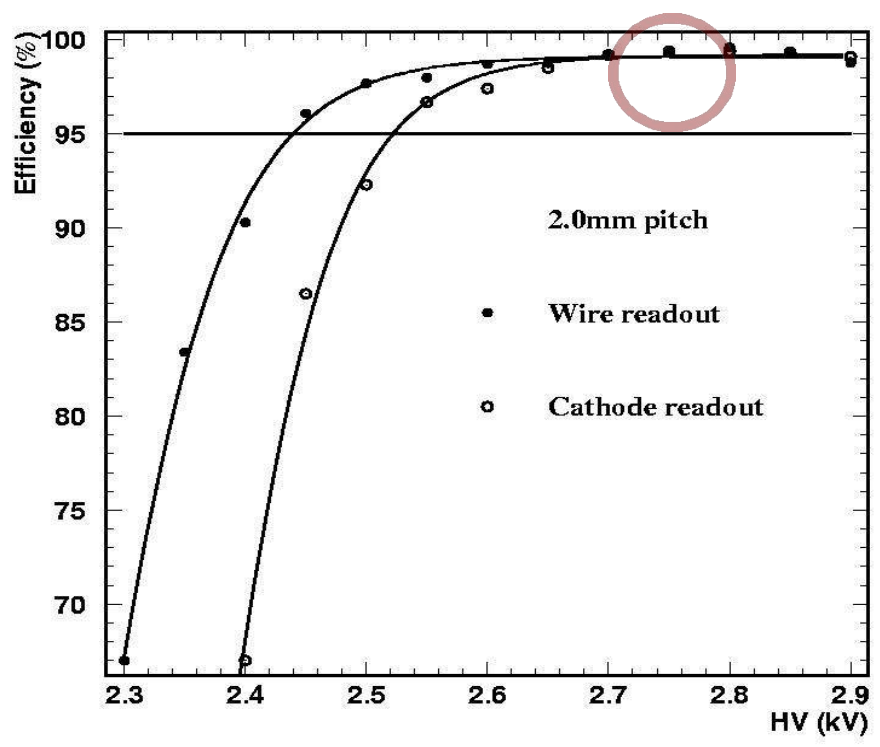

E $\mathrm{M} 1$ radiation length : $0.15 \mathrm{X}_{0}$

(Nomex Honeycomb)
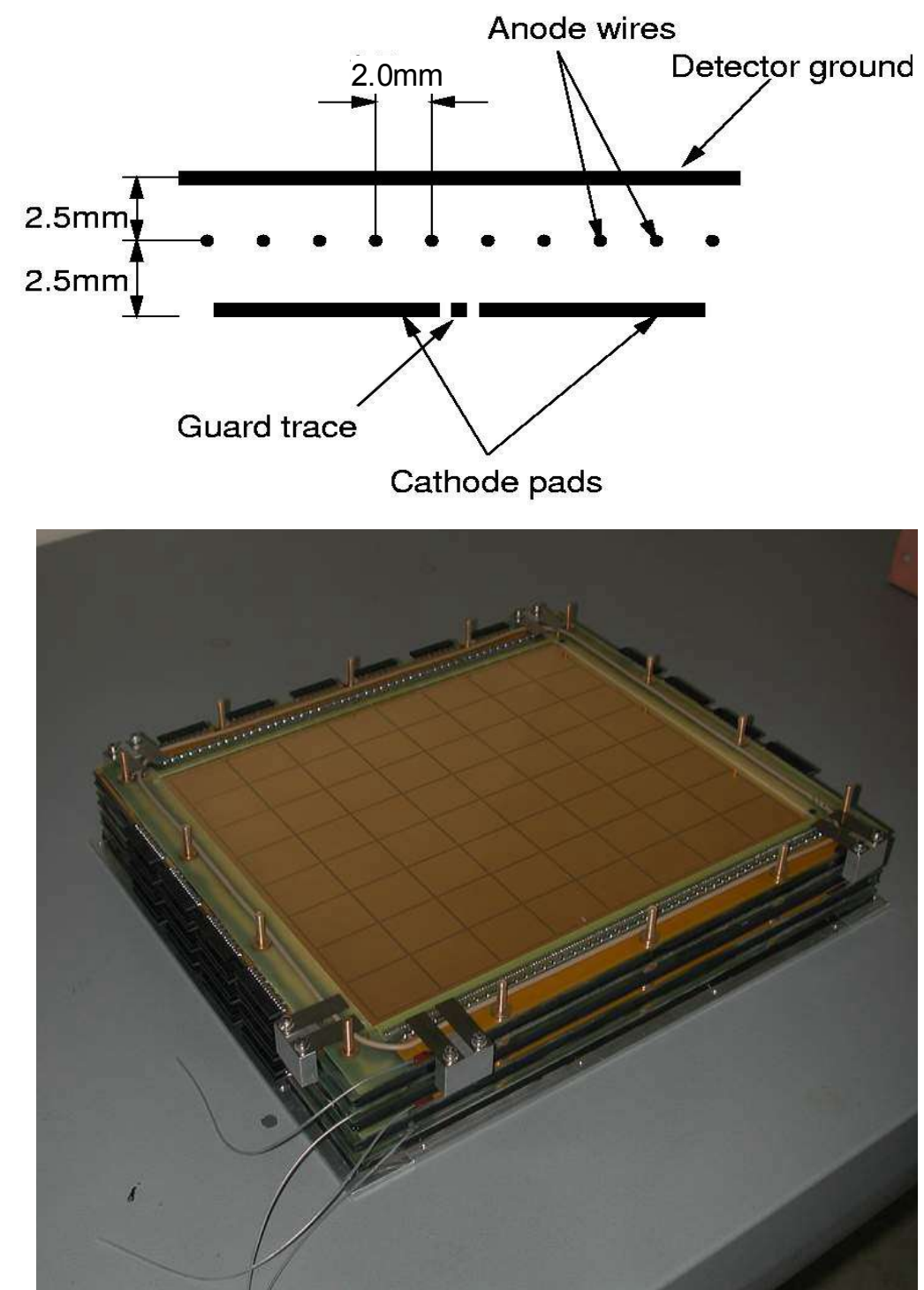

Pre-series MWPC

\section{$\square$ Central Part M1 $\left(0.6 \mathrm{~m}^{2}\right)$ : Triple-Gem ?}




\section{Muon system electronics}

$\square$ Muon electronics Architecture

E Physical Channels : $120 \mathrm{k}$

- Logical pads : $26 \mathrm{k}$

- Made from

- strip crossing

- physical pads

- $\mathrm{L} 0 / \mathrm{L} 1 / \mathrm{DAQ}$

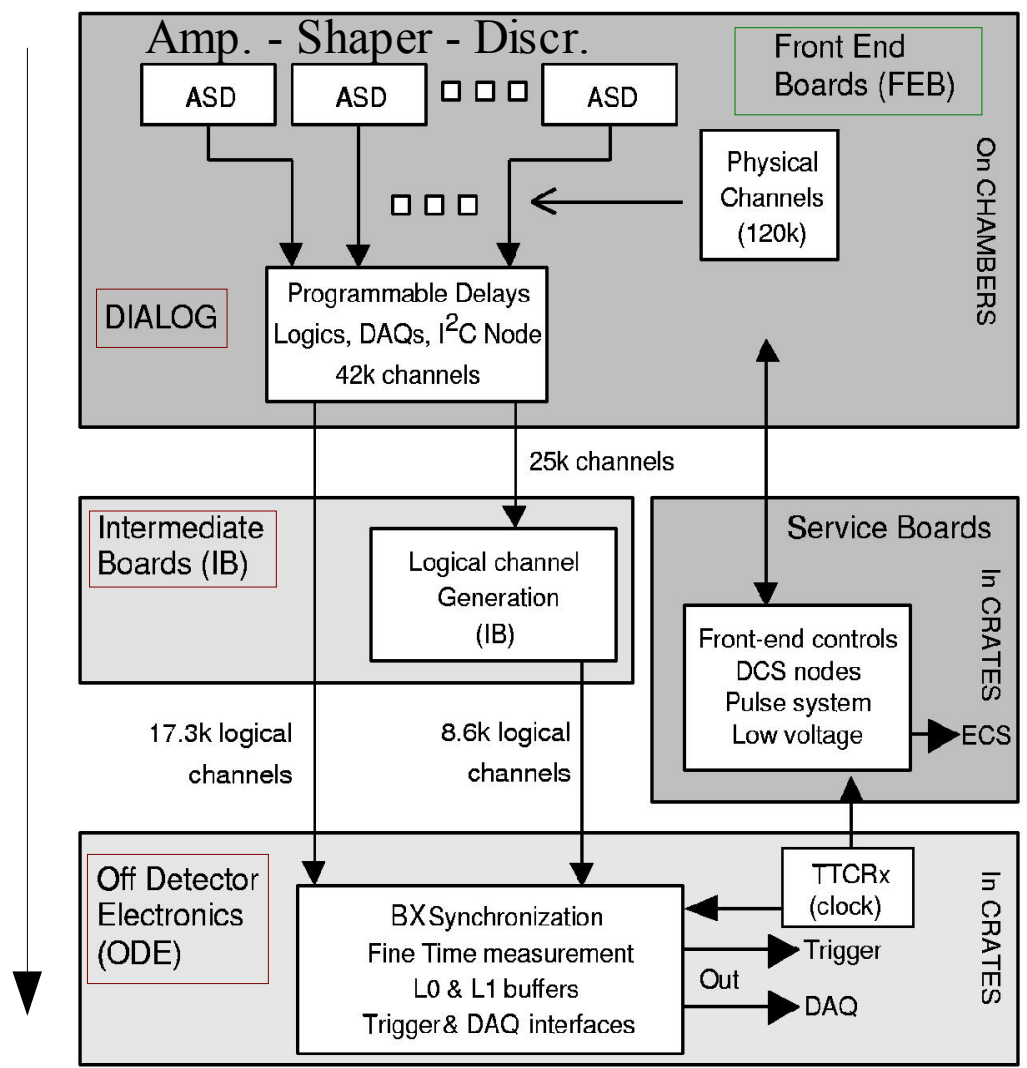

$\square$ MWPC pre-series production started

$\square$ Several sites are ready (tooling and clean room) for production

$\square$ Electronic Architecture has been reviewed and approved

E Final version of most chips has been received 


\section{Lepton identification : Tracking}

\section{$\square$ Tracking performance}

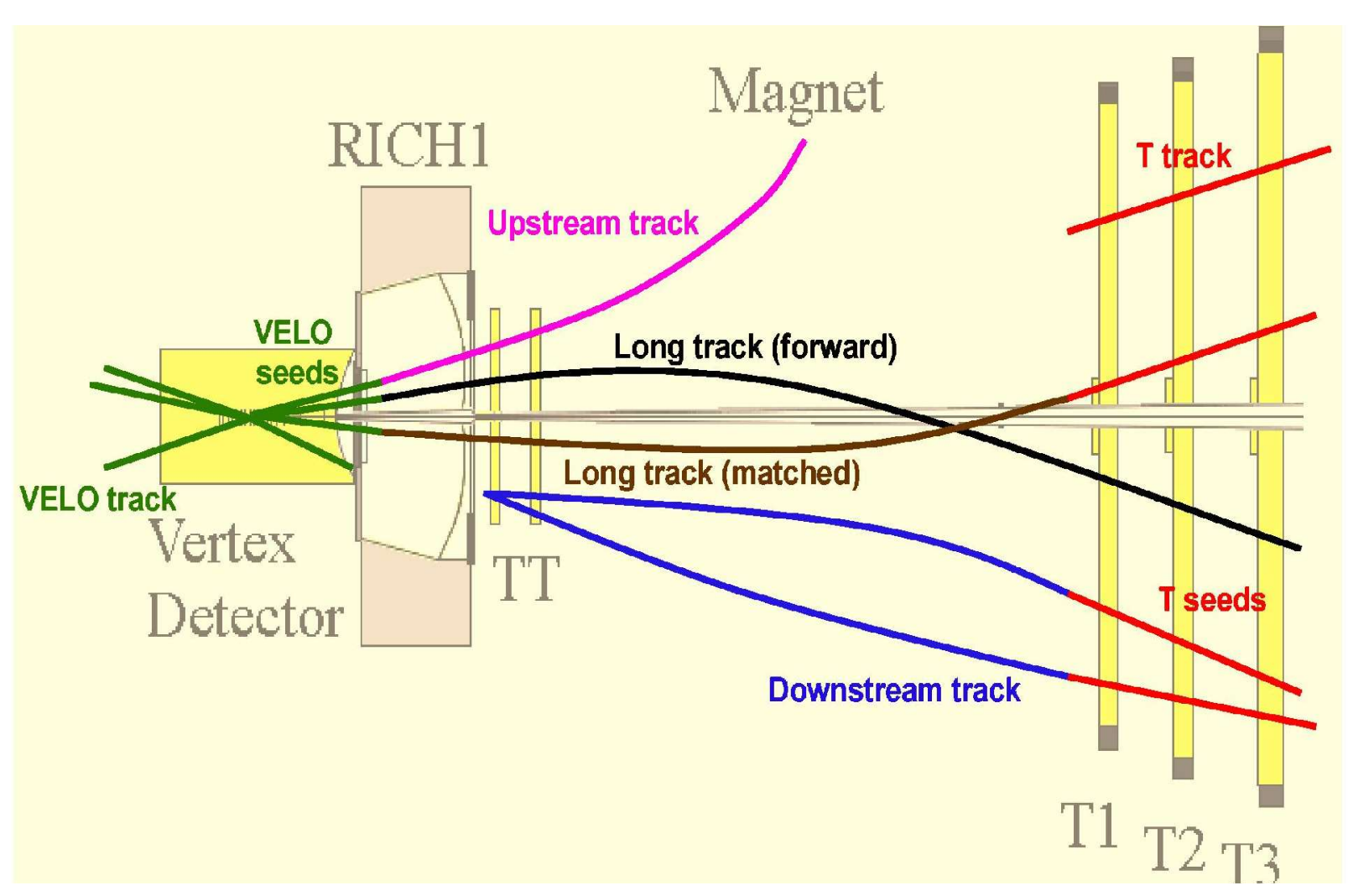

$\square \mathbf{R}_{\text {Ghost }} \sim 3 \%-$ Ptcut $=0.3 \mathrm{GeV}$ 


\section{Electron Identification (I)}

Require impact of a track near a calorimeter cluster

\section{Build 4 parameters :}
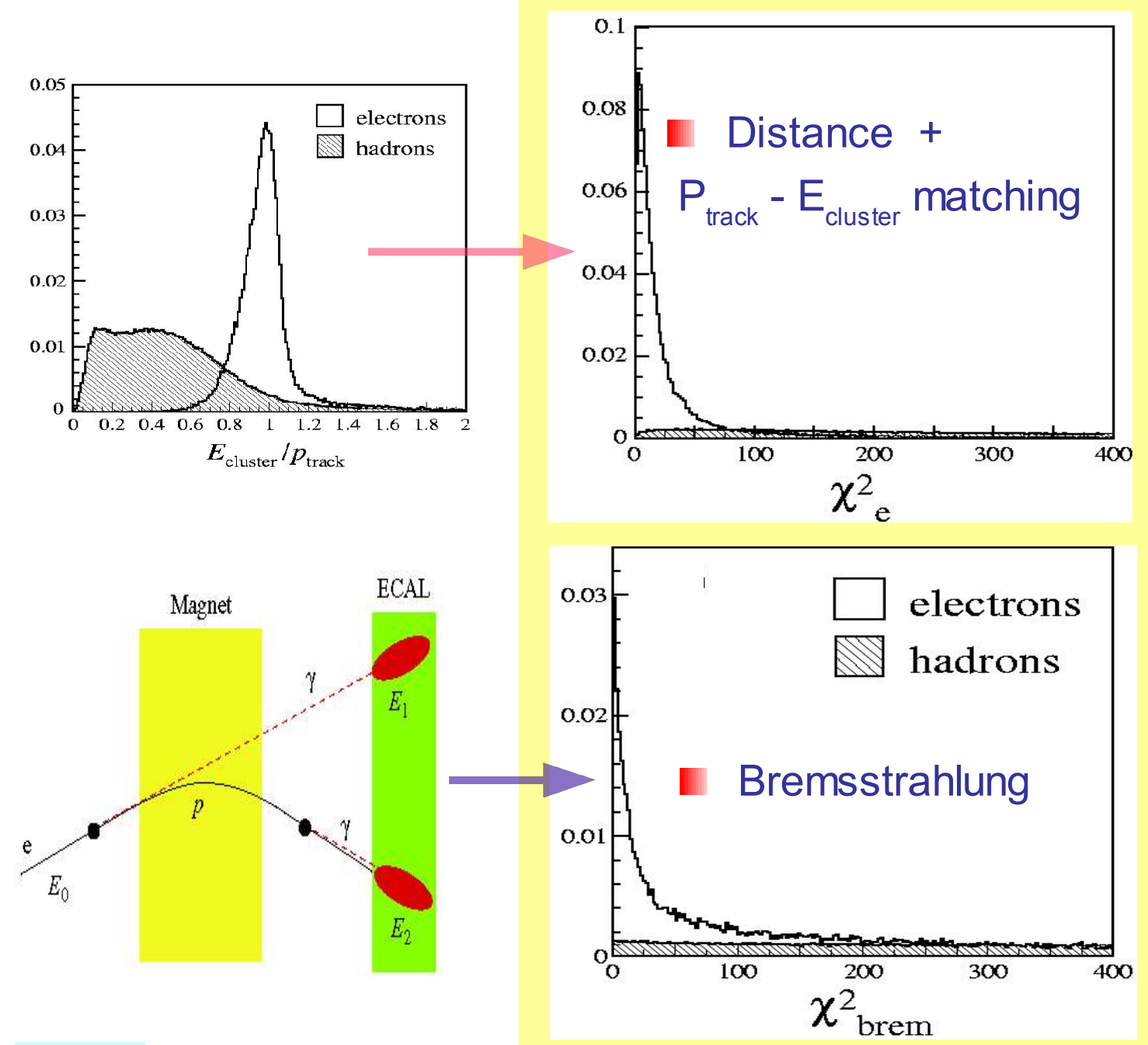
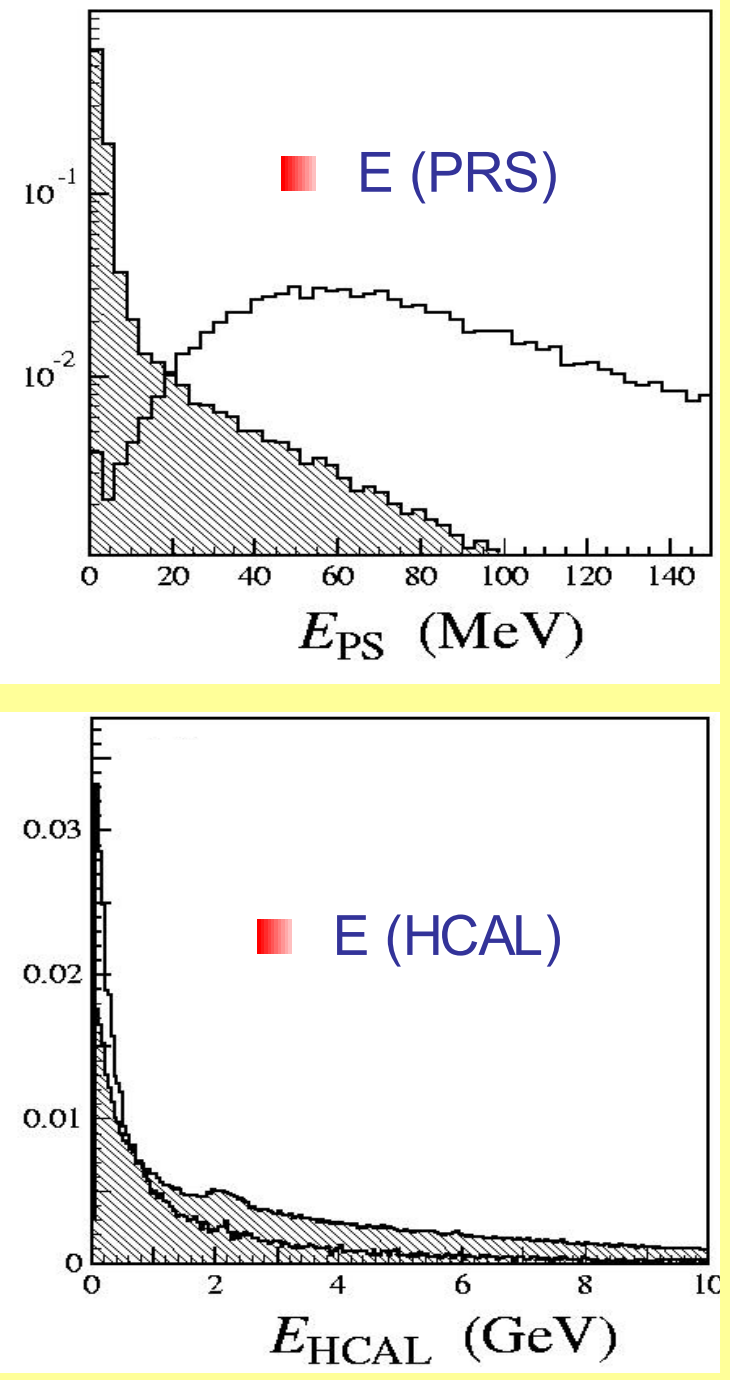


\section{Electron Identification (II) : combined analysis}

Combine Calo + Rich + Muon
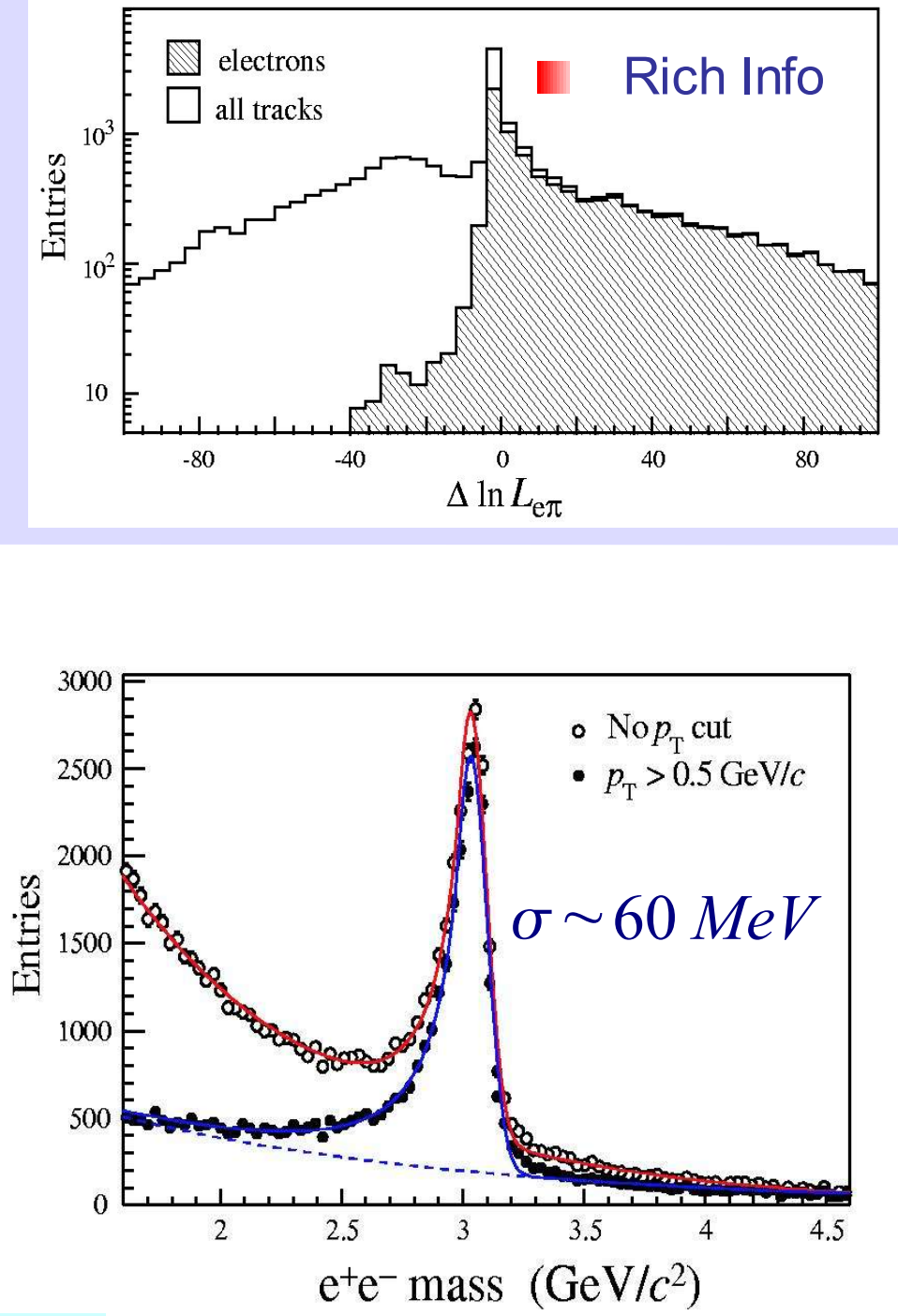

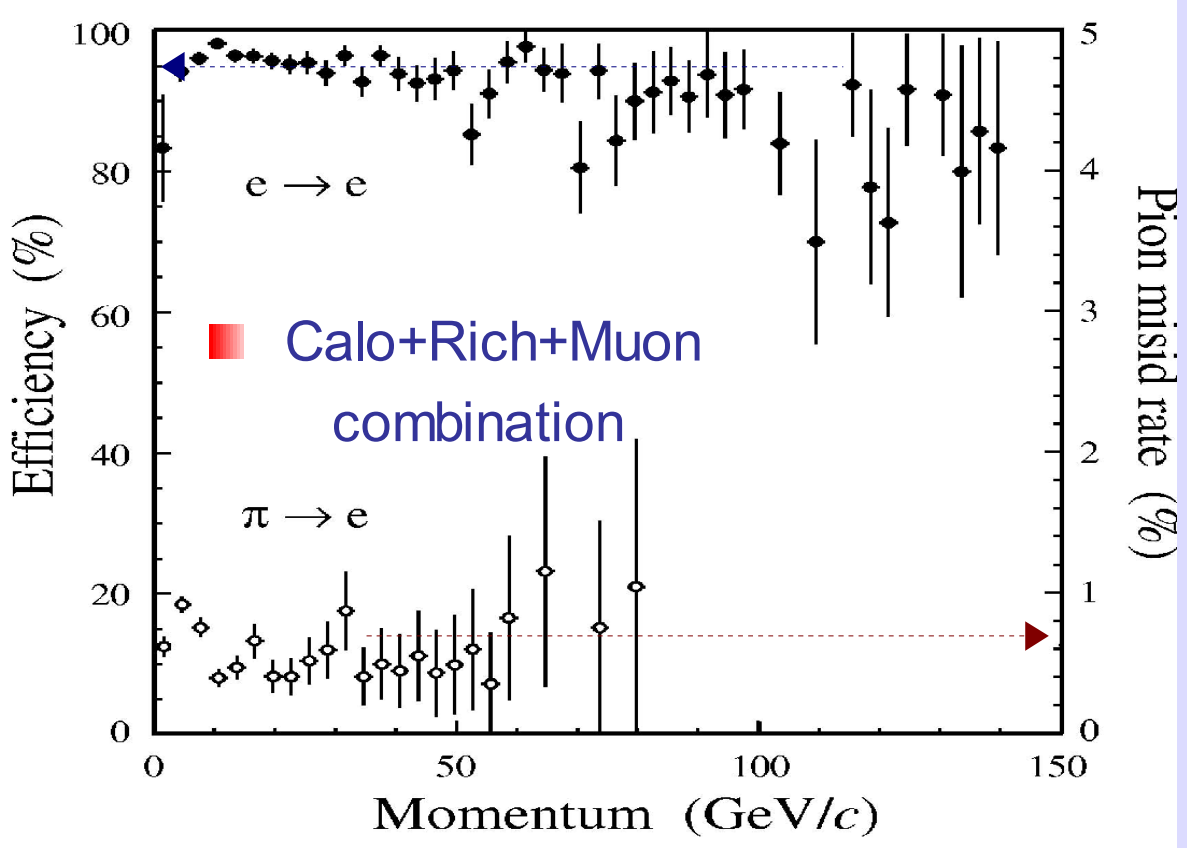

$$
\begin{aligned}
& \mathrm{J} / \Psi \rightarrow \mathrm{e}^{+} \mathbf{e}^{-} \quad\left(\mathrm{B}_{\mathrm{s}}{ }^{0} \rightarrow \mathrm{J} / \Psi \Phi\right) \\
& \text { - } \varepsilon(\mathrm{e})=95 \%-\varepsilon(\Pi \rightarrow \mathrm{e})=0.7 \%
\end{aligned}
$$




\section{Muon Identification (I)}

\section{Definition of Fields of Interest} around track extrapolation

Require hits in \# stations in FOI

$$
\begin{array}{cc}
\text { Momentum }(\mathrm{GeV}) & \text { Muon Stations } \\
3<\mathrm{P}<6 & \mathrm{M} 2+\mathrm{M} 3 \\
6<\mathrm{P}<10 & \mathrm{M} 2+\mathrm{M} 3+(\mathrm{M} 4 \text { or M5 }) \\
\mathrm{P}>10 & \mathrm{M} 2+\mathrm{M} 3+\mathrm{M} 4+\mathrm{M} 5
\end{array}
$$

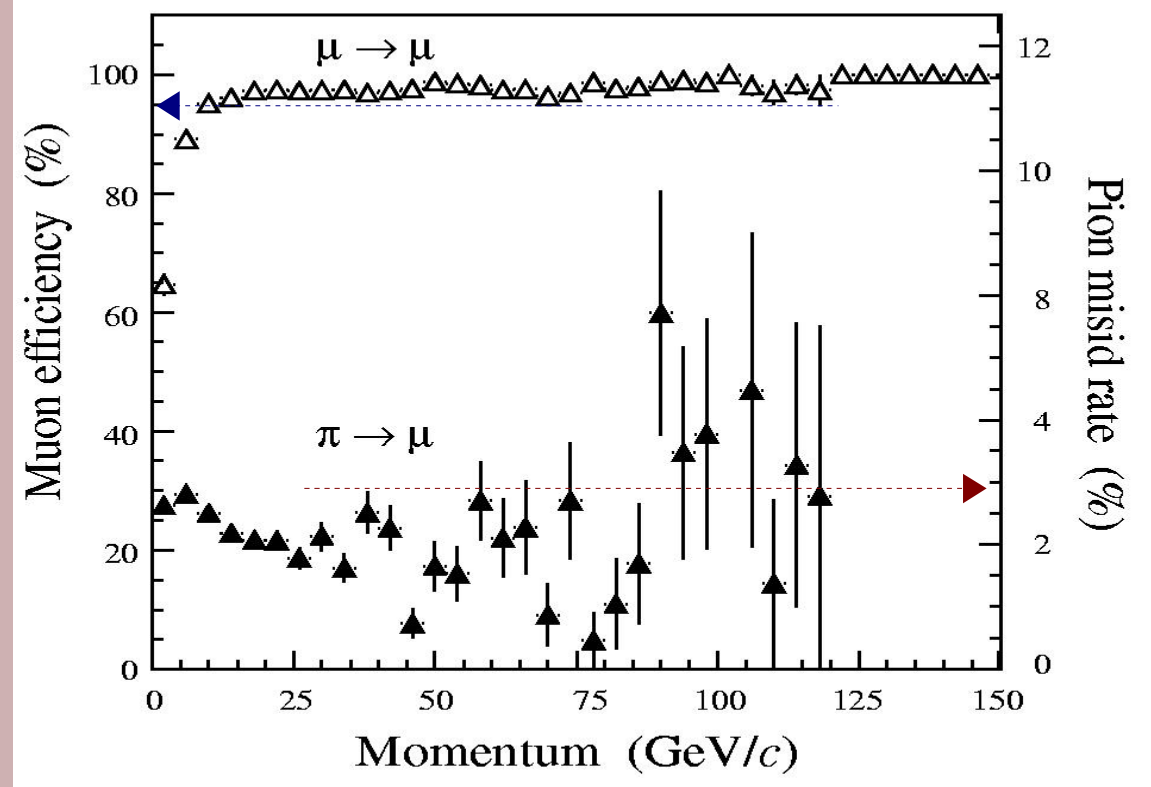

$$
\text { I } \varepsilon(\mu)=94.3 \%-\varepsilon(\pi \rightarrow \mu)=2.9 \%
$$

\section{Estimator to further reject pions}
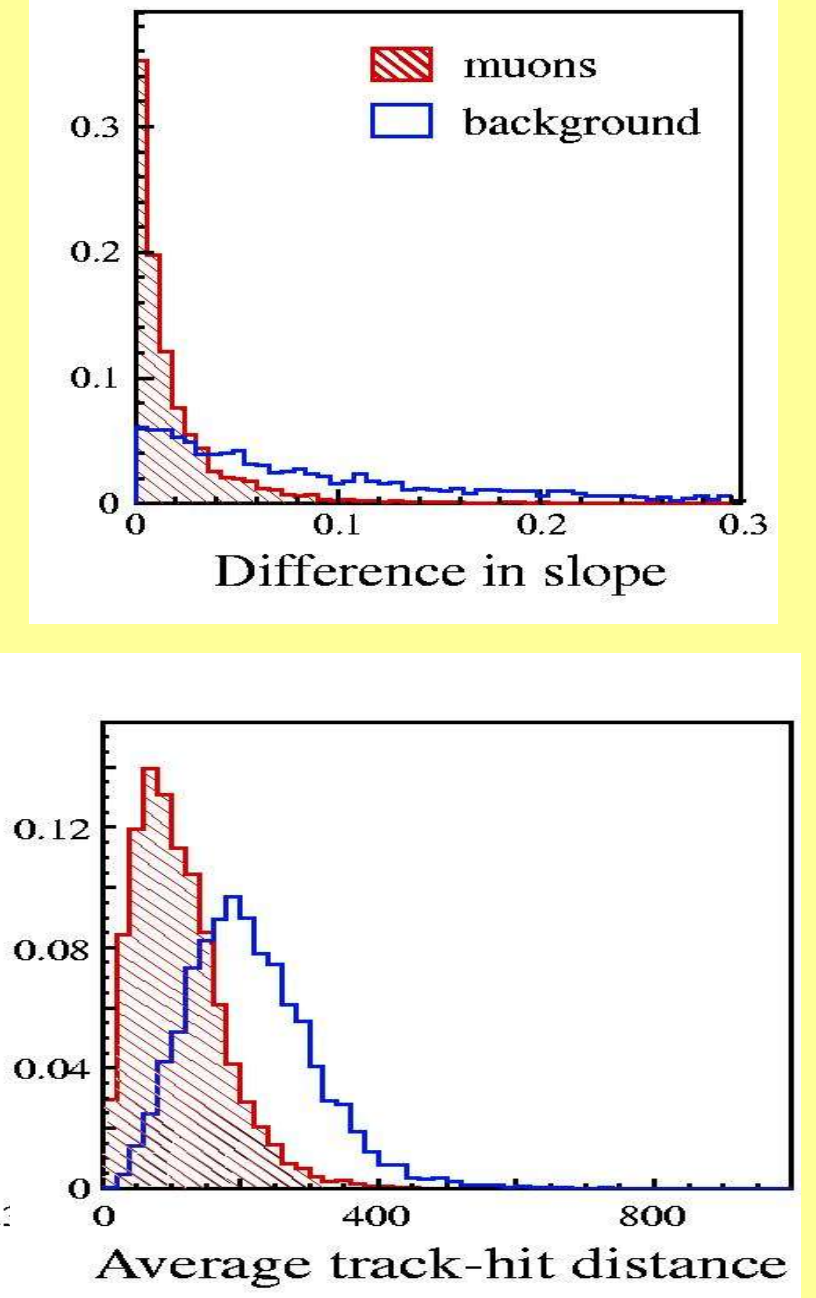


\section{Muon Identification (II)}

\section{Combine Muon+Rich+Calo}

E Further improves selection wrt FOI only

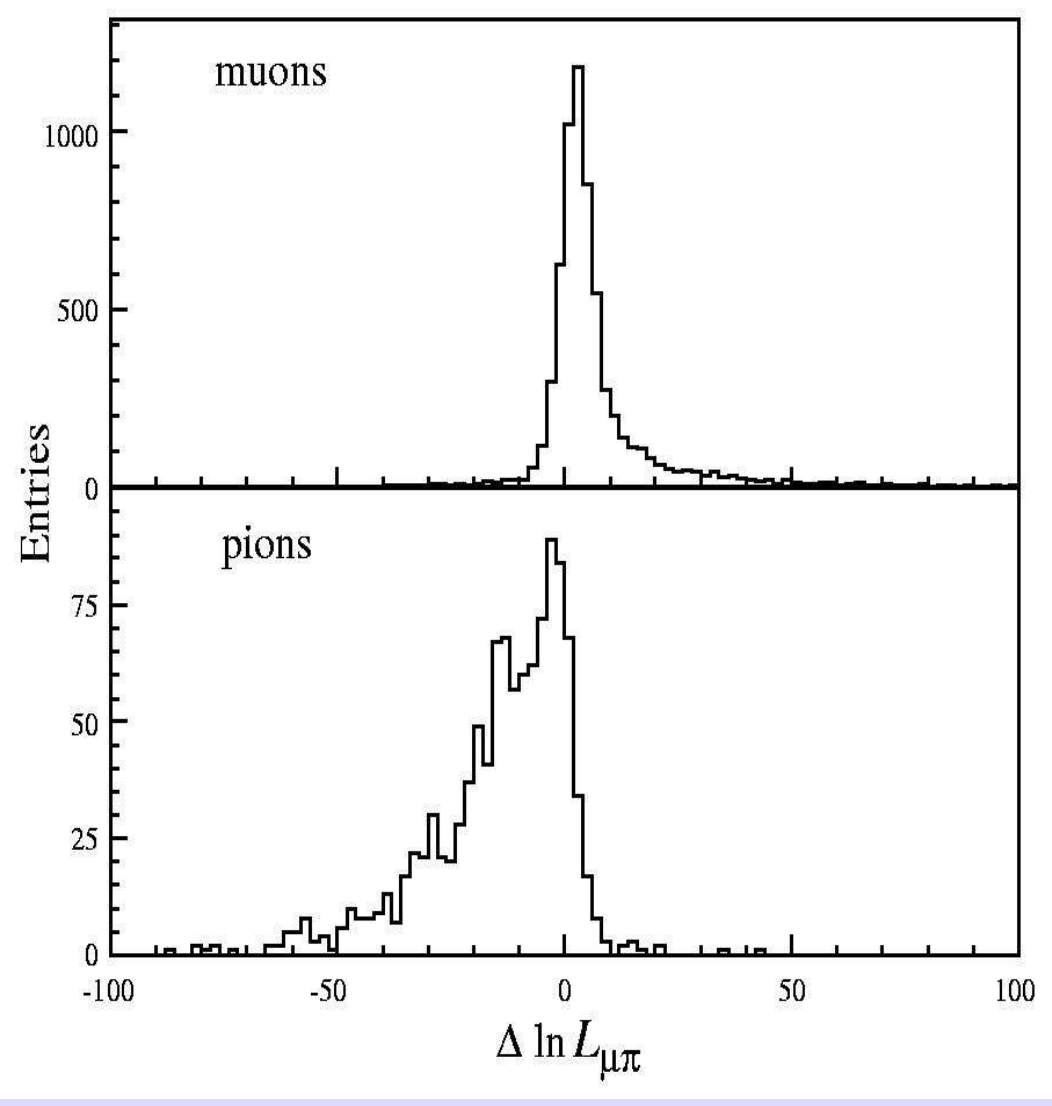

$$
\begin{aligned}
& \mathrm{J} / \Psi \rightarrow \boldsymbol{\mu}^{+} \boldsymbol{\mu}^{-} \quad\left(\mathrm{B}_{\mathrm{s}}{ }^{0} \rightarrow \mathrm{J} / \Psi \Phi\right) \\
& \text { I } \varepsilon(\mu)=93 \%-\varepsilon(\pi \rightarrow \mu)=1 \%
\end{aligned}
$$

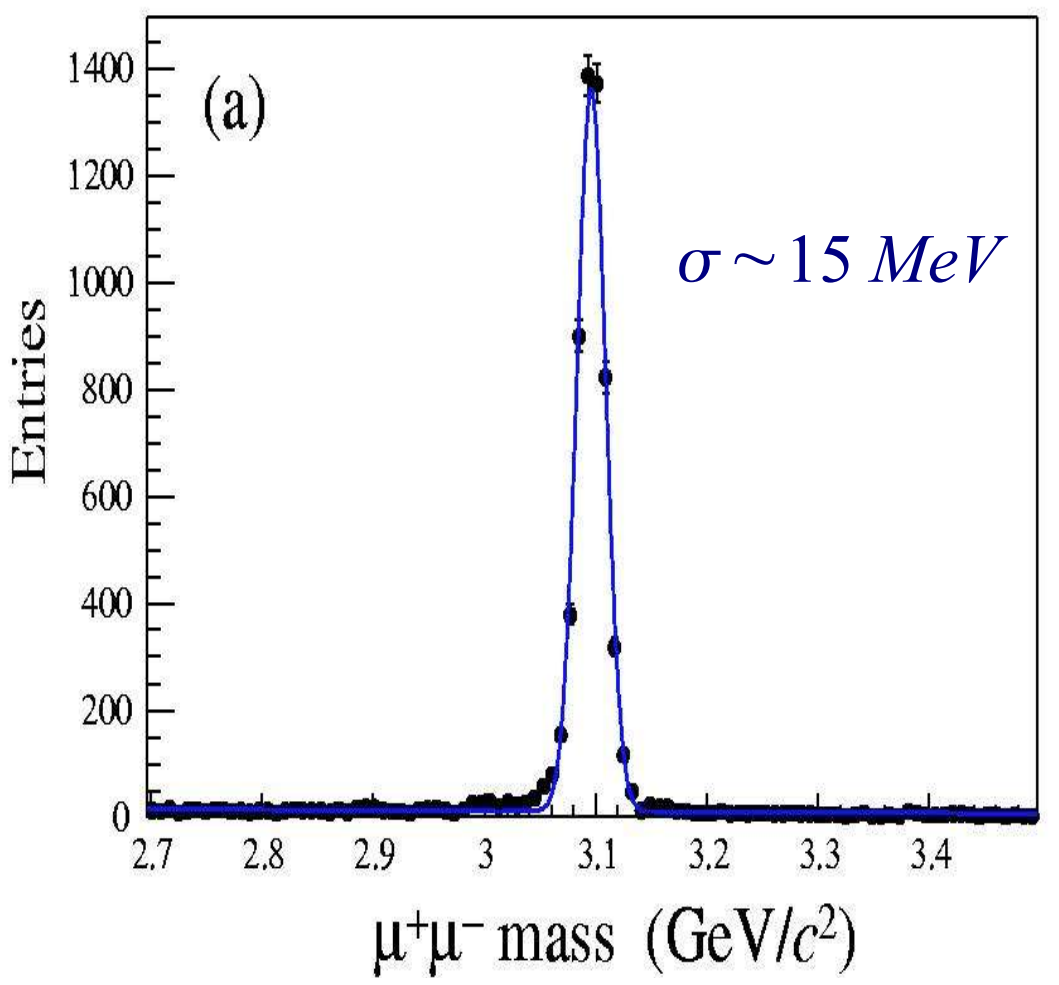




\section{Conclusion}

$\square$ Calorimeter

E Complementary systems

- SPD / PRS / ECAL / HCAL

E LO

E Fast / sensitive / efficient / robust $\square$ Muon system

- Simple (all MWPC) / robust

E LO

- Logical channel : no geometrical ambiguity

Lepton Identification $\left(\mathrm{B}_{\mathrm{s}}{ }^{0} \rightarrow \mathrm{J} / \Psi \Phi\right)$

E Electrons : $\varepsilon(e)=95 \%-\varepsilon(\pi \rightarrow e)=0.7 \%$

- Muons : $\varepsilon(\mu)=93 \%-\varepsilon(\pi \rightarrow \mu)=1 \%$

\section{LHCb will be ready for data taking in 2007 at LHC start-up}

E Detector production is on schedule

E Detector installation starts at the end of next year (magnet installation ongoing) 


\section{Backup Slides}




\section{The LHCb experiment}

\section{$\square$ LHCb is dedicated to the Study of CP violation in the B meson system}

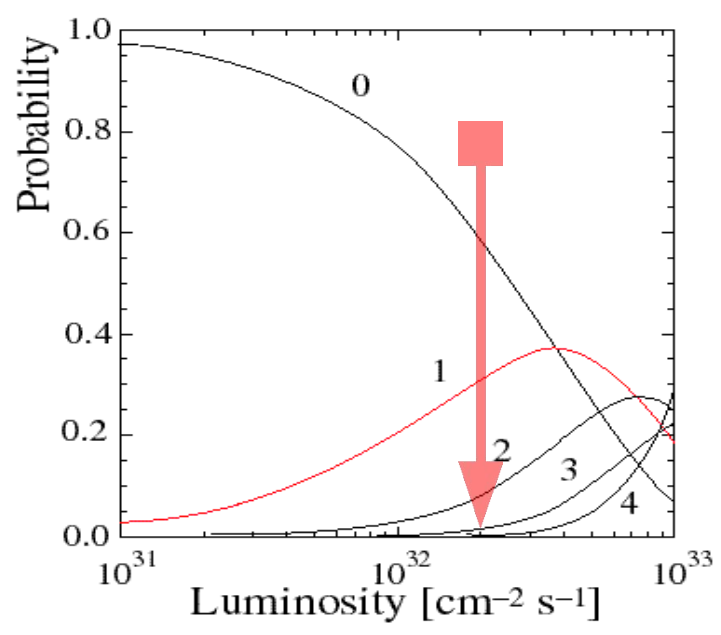

E pp collisions at $\sqrt{ } \mathrm{s}=14 \mathrm{TeV}$

E forward bЂ production correlated

E $\sigma_{\text {total }} \sim 100 \mathrm{mb} \rightarrow$ Int. Rate $: 2 \times 10^{7} \mathrm{~Hz}$

- $\sigma_{\mathrm{bb}} \sim 500 \mu \mathrm{b}, \sigma_{\text {inel }} \sim 80 \mathrm{mb}$

- S/B 1\%

- $10^{12} \mathrm{~b} \overline{\mathrm{b}}$ pairs per year
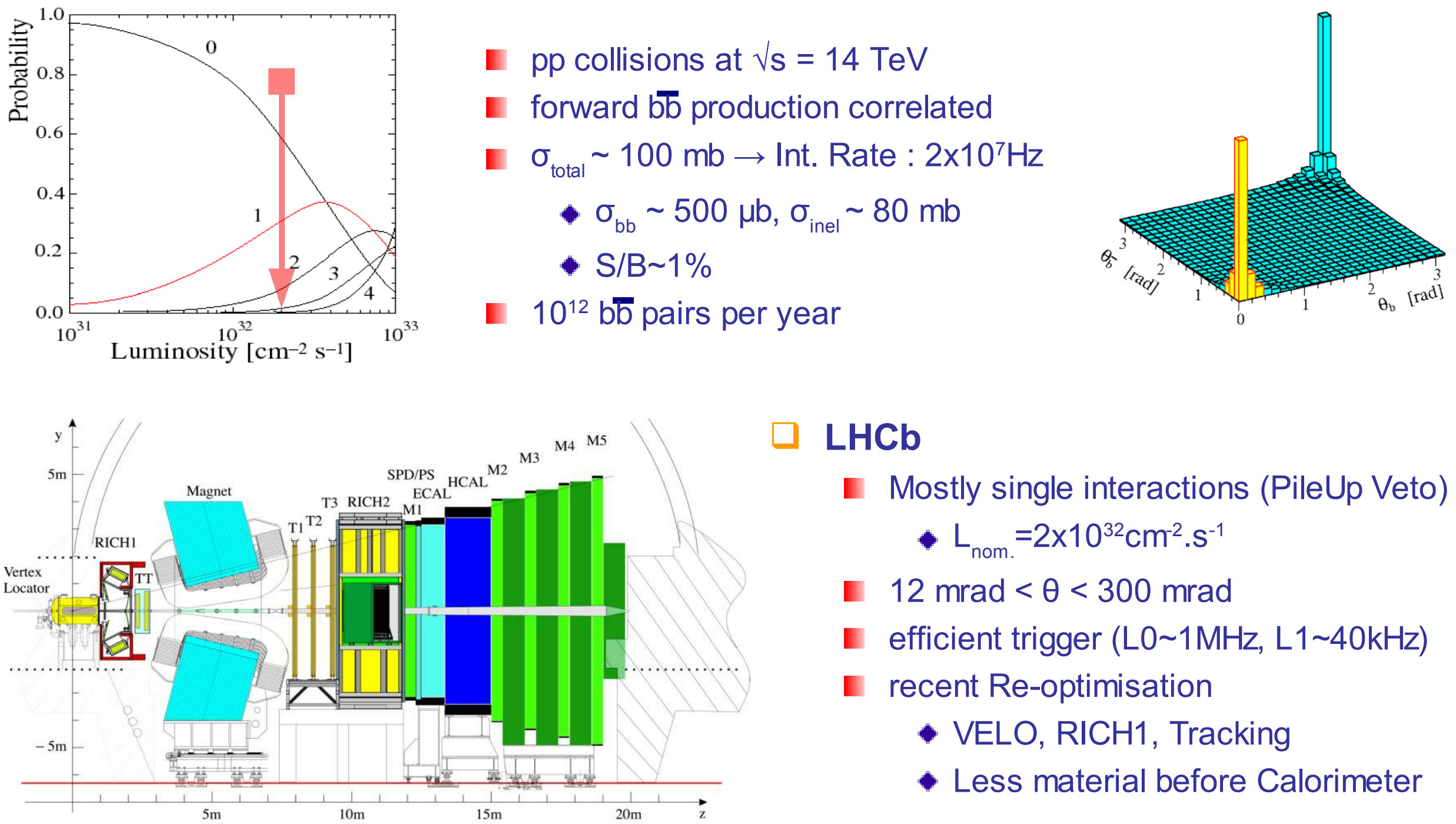

$\square \mathrm{LHCb}$

E Mostly single interactions (PileUp Veto)

- $\mathrm{L}_{\text {nom. }}=2 \times 10^{32} \mathrm{~cm}^{-2} \cdot \mathrm{s}^{-1}$

E $12 \mathrm{mrad}<\theta<300 \mathrm{mrad}$

E efficient trigger (L0 1MHz, L1 40kHz)

- recent Re-optimisation

- VELO, RICH1, Tracking

- Less material before Calorimeter 


\section{Lepton identification : Tracking}

\section{Tracking performance}

E Efficiency

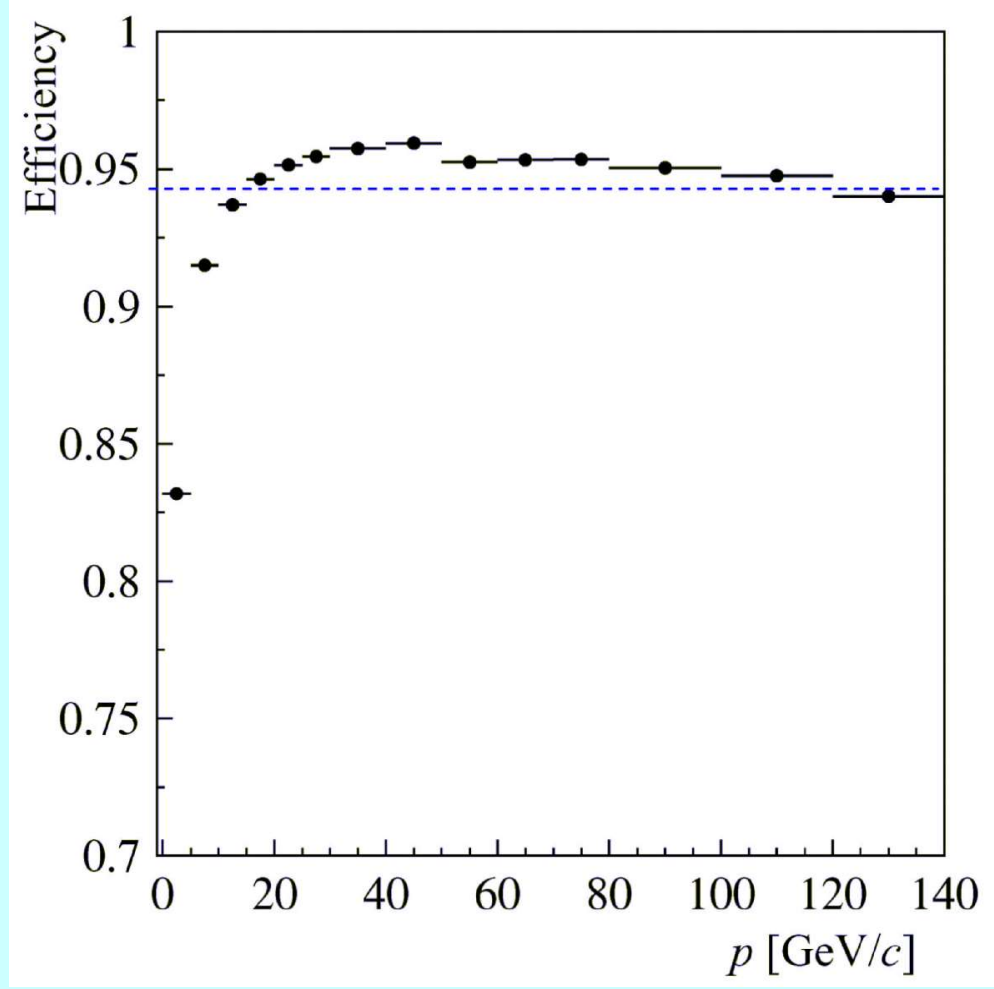

$$
\varepsilon=94 \%-\mathrm{P}>10 \mathrm{GeV}
$$

E Ghost Rate

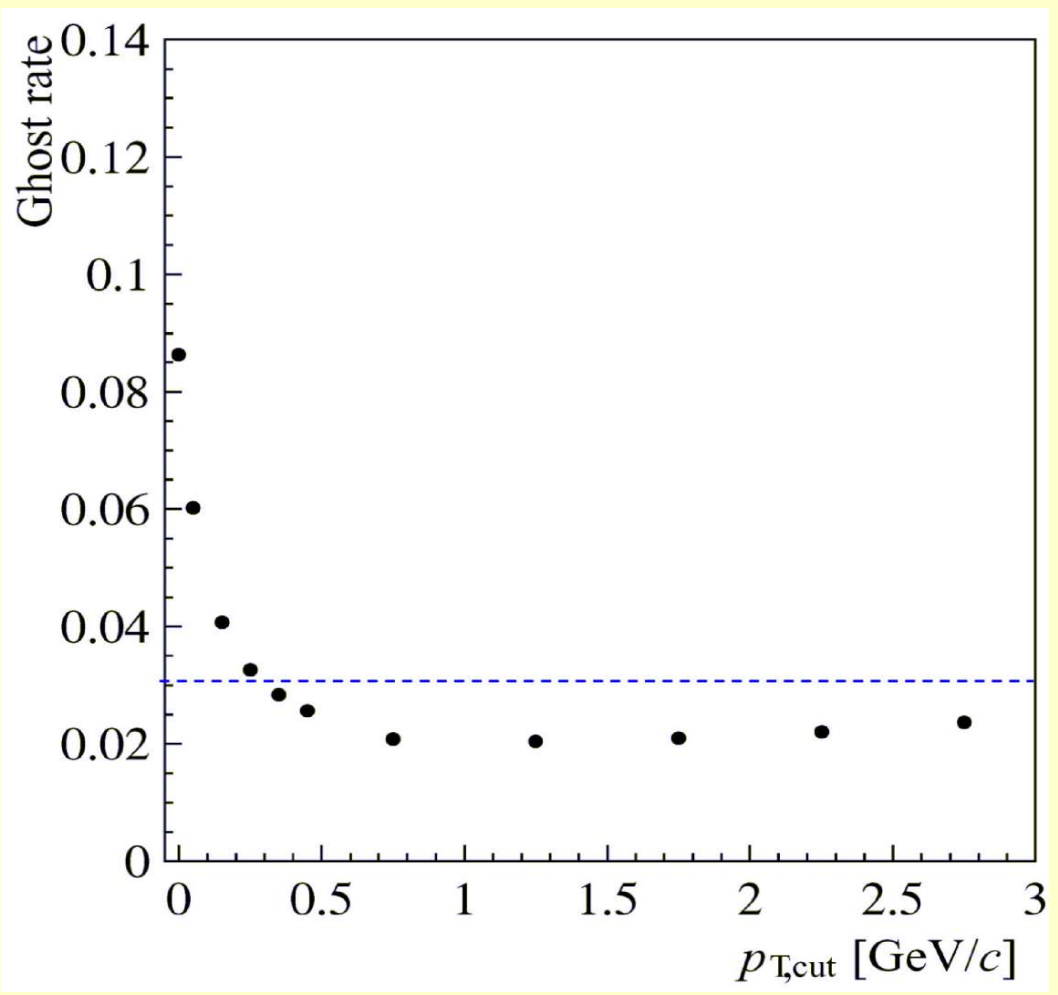

$\square \mathbf{R}_{\text {Ghost }} \sim 3 \%-$ Ptcut $=0.3 \mathrm{GeV}$ 


\section{Lepton Identification Robustness}

$\square$ Electrons (Calo effects)

E Coherent/ incoherent noise increase

- +50 up to $+100 \%$

E Dead channels

- $1 \%(P R S / S P D)-0.2 \%$ (ECAL/HCAL)

E Channel gain error

- $+50 \%$

E No re-tuning (nominal reference histo)

$$
\begin{gathered}
\varepsilon(e) \text { loss of } 2.5 \% \\
\varepsilon(\pi \rightarrow e)=0.7 \% \rightarrow 1 \%
\end{gathered}
$$

$\square$ Electrons (ex. of track multi. Effect)

E Increase track multiplicity by $\mathrm{x} 2$

$$
\begin{gathered}
\varepsilon(e) \text { unaffected } \\
\varepsilon(\pi \rightarrow e)=0.7 \% \rightarrow 1 \%
\end{gathered}
$$

\section{Muons (Muon system effects)}

E Increase low energy background by $x 5$

E No re-tuning

$$
\begin{gathered}
\varepsilon(\mu)=94 \% \text { unaffected } \\
\varepsilon(\pi \rightarrow \mu)=2.9 \% \rightarrow 11.7 \%
\end{gathered}
$$

E After algorithm re-tuning

$$
\begin{gathered}
\varepsilon(\mu) \text { loss of } 7 \% \\
\varepsilon(\pi \rightarrow \mu) \text { back to } 2.9 \%
\end{gathered}
$$

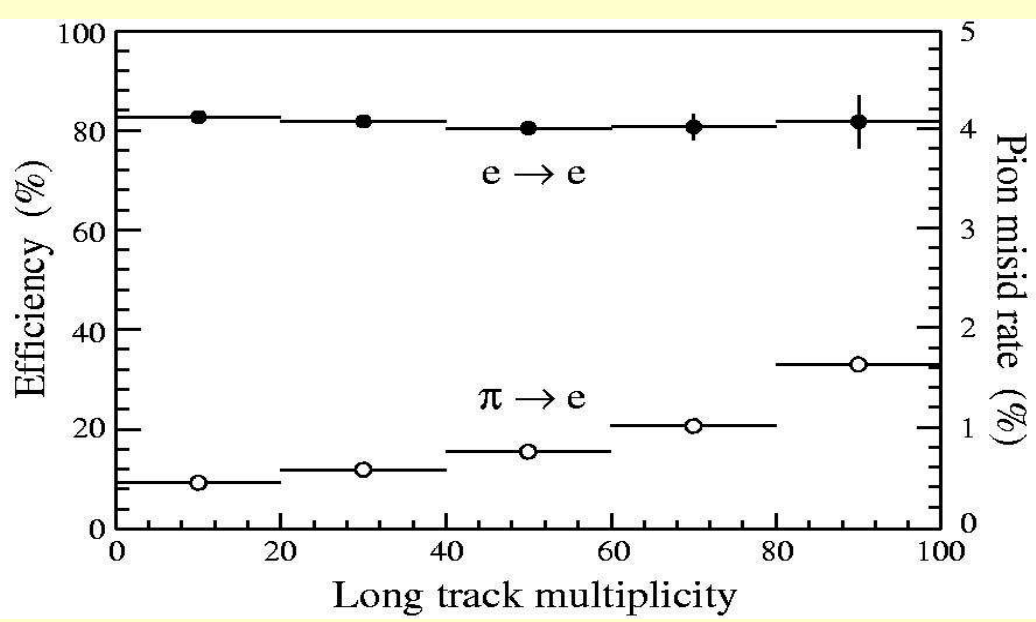

